\title{
Using Game Theoretic Models to Predict Pilot Behavior in NextGen Merging and Landing Scenario
}

\author{
Yildiray Yildiz* \\ U. C. Santa Cruz, Moffett Field, California, 94035, USA \\ Ritchie Lee $^{\dagger}$ \\ Carnegie Mellon University, Moffett Field, California, 94035, USA \\ Guillaume Brat ${ }^{\ddagger}$ \\ Carnegie Mellon University, Moffett Field, California, 94035, USA
}

\begin{abstract}
In this paper, we present an implementation of the "Semi Network-Form Game" framework to predict pilot behavior in a merging and landing scenario. In this scenario, two aircraft are approaching to a freeze horizon with approximately equal distance when they become aware of each other via an ADS-B communication link that will be available in NextGen airspace. Both pilots want to gain advantage over the other by entering the freeze horizon earlier and obtain the first place in landing. They re-adjust their speed accordingly. However, they cannot simply increase their speed to the maximum allowable values since they are concerned with safety, separation distance, effort, possibility of being vectored-off from landing and possibility of violating speed constraints. We present how to model these concerns and the rest of the system using semi network-from game framework. Using this framework, based on certain assumptions on pilot utility functions and on system configuration, we provide estimates of pilot behavior and overall system evolution in time. We also discuss the possible employment of this modeling tool for airspace design optimization. To support this discussion, we provide a case where we investigate the effect of increasing the merging point speed limit on the commanded speed distribution and on the percentage of vectored aircraft.
\end{abstract}

\section{Nomenclature}

$\mathrm{A}_{\mathrm{i}} \quad i^{\text {th }}$ Aircraft

$v_{i}^{0} \quad$ The initial velocity of $\mathrm{A}_{\mathrm{i}}$

$v_{i}^{c} \quad$ The new velocity of $\mathrm{A}_{\mathrm{i}}$ commanded by the pilot

$x_{i} \quad$ The initial distance of $\mathrm{A}_{\mathrm{i}}$ to the merging point

$d_{i} \quad$ The final distance of $\mathrm{A}_{\mathrm{i}}$ to the merging point

\section{Introduction}

Human decision-makers play a major role in the operation of most real-world systems of today. In most cases, the successful operation of these systems often hinge upon the sound judgement of few individuals. For example, pilots and air traffic controllers continuously make decisions that determine the safety and operation of the National Airspace System (NAS). Even if replacing the humans with automation is conceivable, it will be many decades before the dependence on human decision-making becomes negligible. Since humans play

*Associate Scientist, University Affiliated Research Center, NASA Ames Research Center MS 269-1, Moffett Field, CA, AIAA Senior Member.

$\dagger$ Robotics Researcher, Silicon Valley Campus, NASA Ames Research Park MS23-11, AIAA Member.

${ }_{\ddagger}^{\ddagger}$ Technical Lead, Silicon Valley Campus, NASA Ames Research Center, MS 269-1, AIAA Senior Member. 
such a crucial role in characterizing real-world systems, it follows that to make any accurate predictions about system behavior requires a model that is capable of capturing both the human and non-human dynamics of the system.

Combining ideas from game theory and computer science, the "Semi Network-Form Game" ${ }^{1}$ framework can be used not only to model multiple human decision-makers and physical systems, but also to capture their interactions. For example in their paper, ${ }^{1}$ the authors used the framework to model pilot behavior during mid-air encounters. A semi network-form game (or "semi net-form game") uses a specific type of probabilistic graph, called a Bayesian network, ${ }^{2}$ as the underlying probabilistic framework, consequently representing all parts of the system using random state variables. Non-human components such as automation and physical systems are described by traditional probability nodes (called "chance nodes"), i.e., nodes that have their conditional probability distributions pre-specified. Human decisions are represented by special nodes (called "decision nodes") that have their conditional probabilities left unspecified. Instead, these nodes are each associated with a utility function, ${ }^{3}$ i.e., a function that summarizes the preferences of a decision-maker into a single "happiness" metric. The notion is that humans do not act according to a fixed conditional probability distribution, but rather choose their actions in attempt to maximize their utility. However, one difficulty arises when there are multiple competing decision-makers. How do we decide whether a single utility is maximized or if there is a compromise? To answer this question, semi net-form games turn to the game theory literature, where researchers have found simple mathematical models, called "solution concepts", that accurately modeled interacting human behavior in past experiments. ${ }^{4-6}$ Combining Bayes nets, solution concepts, and statistical techniques, Lee and Wolpert created an algorithm, called "Level-K d-relaxed strategies", ${ }^{1}$ that efficiently estimates the behavior of a semi net-form game model.

LibNFG is a NASA open source software library that implements the semi net-form game framework and its estimation algorithms. The software, written in $\mathrm{C}++$, allows the user to specify all aspects of a semi net-form game model including topology, node behavior, and utility functions, and computes sample estimates of the system behavior. In this paper, we demonstrate how to apply a semi net-form game to model a merging and landing scenario set in a future airspace, and analyze the prediction results. In addition, we discuss how this approach can be used to optimize airspace scenarios. As an example, we provide quantitative predictions on how relaxing merging point speed constraint effects the number of vectored aircraft and the final distance between the aircraft, in the merging and landing scenario. These kind of predictions can be utilized in an optimization routine by the system designer to achieve optimum system parameters that will satisfy the design goals and constraints.

\section{Merging and Landing Scenario as a Semi Net-Form Game}

In the existing system, aircraft are controlled through different sectors by various ground controllers, until they arrive at the "freeze horizon". After entering the freeze horizon, the aircraft become eligible for sequencing by the airport ground controller based on a "first come first serve" (FCFS) principle. ${ }^{7}$ In the Next Generation (NextGen) airspace, the aircraft are supposed to have ADS-B data-link, via which they will be able to receive other aircraft information such as aircraft ID, position and velocity. In the scenario we want to investigate, we assume that two aircraft are converging to the freeze point and they each realize, via ADS-B, that there is another aircraft at an approximately equal distance to the freeze horizon (see Fig. 1). Since they are aware of the FCFS procedure, they would like to use this new ADS-B technology to obtain the first place in the landing sequence without creating a safety hazard.

\section{A. Scenario Description}

We model the merging and landing scenario as follows:

1. At time equal to $t_{0}$, the aircraft have velocity $v_{i}^{0}$ and position $x_{i}$.

2. At time equal to $t_{0}$, pilots realize that there is another aircraft close to the freeze horizon and they simultaneously decide (and act) to adjust their speed to $v_{i}^{c}$.

3. When the new velocity $v_{i}^{c}$ is commanded to the flight controller, the aircraft reaches to this velocity through a first order dynamics.

4. The ground controller first observes these two aircraft on her radar screen at time $t_{0}+t_{\text {travel }}$ 


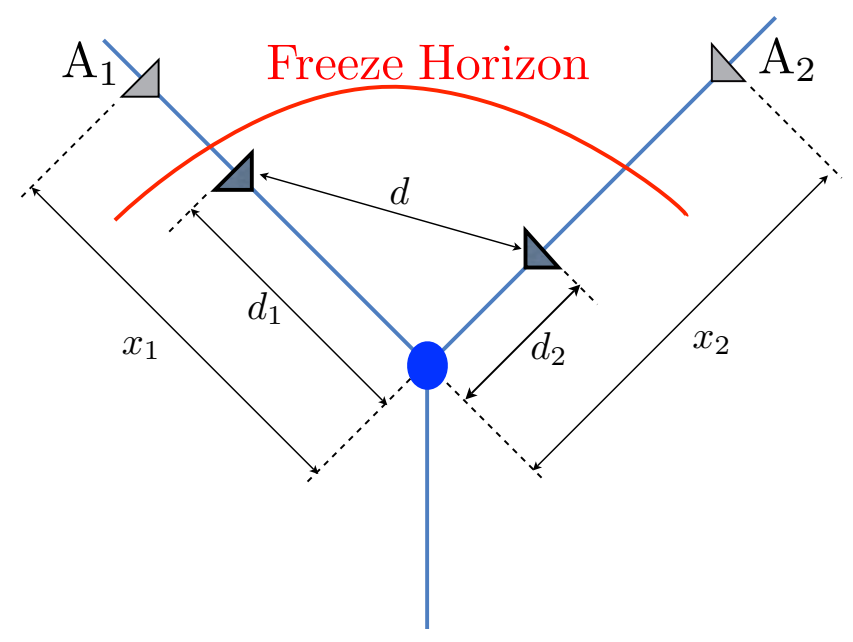

Figure 1. Two aircraft converging to the freeze horizon

5. When the ground observes the aircraft, she determines whether or not to vector the aircraft off from landing. The ground deterministically decides whether to vector-off $(V O F F=1)$ or not $(V O F F=0)$ based on the following rule:

$$
V O F F_{i}=\left\{\begin{array}{cc}
1 & \text { if } \quad-t^{*}<\left(\frac{d_{i}}{v_{i}^{c}}-\frac{d_{j}}{v_{j}^{c}}\right)<c t^{*} \\
0 & \text { otherwise }
\end{array}\right.
$$

where $c$ and $t^{*}$ are a known constant and a threshold time value.

It is noted that in (1), the expression inside the parenthesis is the difference between the estimated time of arrivals (ETA) of the Aircraft to the merging point when they are first observed by the ground. According to this rule, if the ETA of $\mathrm{A}_{i}$ to the merging point is close to the ETA of the other aircraft so that $\left|\mathrm{ETA}_{i}-\mathrm{ETA}_{j}\right|<t^{*}$ then it is vectored regardless of whether it has a higher ETA or not. However, if $A_{i}$ manages to get ahead more than $t^{*}$ seconds, then it will not be vectored off. On the other hand, if it falls behind only so much so that $c t^{*}>\left(\mathrm{ETA}_{i}-\mathrm{ETA}_{j}\right)>t^{*}$ it will still be vectored off. So the pilots should strategize in such a manner that they should keep their ETA different from the other aircraft but if they have to keep it close, then they should be a certain time seconds ahead not to be vectored off.

It is assumed that the merging lines in Fig. 1 are orthogonal and therefore the distance between the aircraft when they are first observed by the ground can be calculated as

$$
d=\sqrt{d_{1}^{2}+d_{2}^{2}} .
$$

\section{B. Graphical Model}

The graphical model, shown in Fig. 2, specifies the relationships between system variables of the merging and landing scenario. In this figure, the true state of Aircraft $1,\left[x_{1}, v_{1}^{0}\right]$ is represented by node $S_{1}$ and the true state of Aircraft 2, $\left[x_{2}, v_{2}^{0}\right]$ is represented by node $S_{2}$. These are initialized from a uniform distribution with ranges [220 - 270] in nautical miles and [340-420] in knots, along their trajectories. Although the pilots know their own aircraft's state, they observe the other aircraft's state through a noisy observation node. The observations obtained through ADS-B data-link are represented by the nodes $W_{i}^{\text {ADS-B }}$, which will be the same as the true states but with additive observation noise. Pilots, $\mathrm{P}_{i}$, use these observations, together with their beliefs about the other pilot's and the ground's decisions, to decide new speed commands $v_{i}^{c}$ to the autopilot controllers. Using the true states and pilot's moves, the aircraft motion is then simulated to 


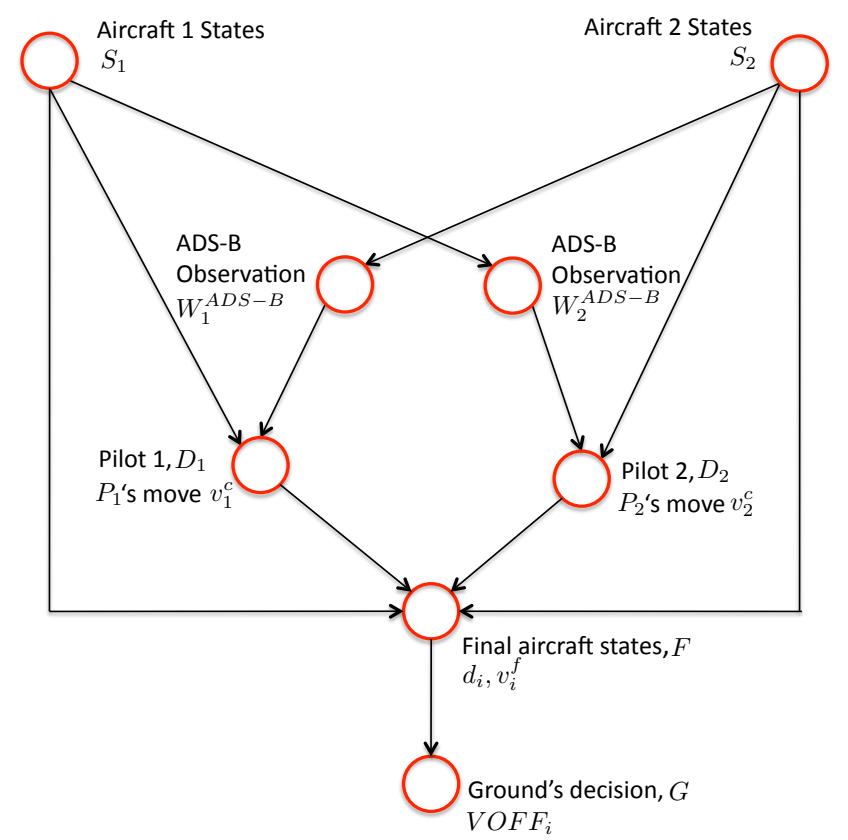

Figure 2. Bayes net diagram of a merge and land scenario

obtain the outputs, $\left[d_{i}, v_{i}^{f}\right]$, which is represented by the node $F$. Finally, the ground observes the resulting aircraft states and decides whether to vector off an aircraft $(V O F F=1)$ or not $(V O F F=0)$.

\section{Aircraft Model}

The aircraft in this scenario is being controlled by the auto-pilot which is in velocity control mode. In this mode, the pilot command the velocity $v^{c}$ and the autopilot drives the aircraft to the desired speed. It is assumed that the closed loop system which has the pilot command as the input and the aircraft velocity as the output is represented with a first order dynamics that has a 33-second time constant. This time constant is obtained from NASA's Generic Transport Model (GTM) which represents a generic large transport aircraft together with a conventional autopilot. Therefore, the aircraft speed change can be given by the following equation.

$$
\dot{v}_{i}=-\frac{1}{33} v_{i}+\frac{1}{33} v_{i}^{c}
$$

The aircraft are assumed to be perfectly following the merging path lines and therefore only the motion on these lines are considered. Therefore, the relation between the initial positions $x_{i}$ and the final positions $d_{i}$ can be given as

$$
d_{i}=x_{i}-\int_{t_{0}}^{t_{0}+\Delta t} v_{i}(\sigma) \mathrm{d} \sigma
$$

where $\Delta t$ is assumed to be 900 seconds.

\section{Pilot Utility Functions}

Pilot utility functions summarize pilot preferences about different states of the system into a single metric. For the merging and landing scenario, it is assumed that the following factors plays a role in pilots' decisions:

Estimated Time of Arrival: Pilots want to arrive to the airport as soon as possible. To achieve this goal, they try to minimize their estimated time of arrival which is roughly proportional to the distance $d_{i}$ of the aircraft to the merge point when they are first observed by the ground. 
Aircraft Separation: Pilots want to prevent an unsafe situation and therefore they want to keep the separation between each other as much as possible. Therefore, they try to maximize $d$ (see Fig. 1).

Possibility of Being Vectored Off: Pilots do not want to be vectored-off from landing because it delays their arrival, causes extra fuel burn and requires additional effort and inconveniences. Thus the pilot will choose actions that try to make the ground decide against vectoring-off. The variable that represents vectoring off decision is $V O F F$, which takes the values 0 and 1 , representing "not vectoring off" and "vectoring off", respectively.

Effort: If possible, pilots do not want to do anything and keep their speed unchanged to minimize effort. Therefore, they will try to minimize $\left|v^{0}-v^{c}\right|$.

Violating the Merging Point Constraint: The pilots are supposed to have a certain speed $v^{*}$ at the merging point. Therefore, the more they deviate from that speed the harder it will be to satisfy the constraint in the remaining time after they are first observed by the ground. Since the environment is uncertain, it is not clear what the "maximum safe speed" is while passing through the freeze horizon. Therefore, pilots try to adjust their speed as close as possible to $v^{*}$, without having a clear idea how much they can deviate from it safely. As the deviation magnitude increases, the probability of violating the constraint increases, which increases the nervousness of the pilots. However, increasing the speed beyond a certain value will have negligible additional effect. We will model this dependency using a hyperbolic tangent function with certain constants to have it positive everywhere and to represent reasonable speed ranges.

Summing all the above mentioned terms, the pilot utility function is given as

$$
U_{i}=-\omega_{1} d_{i}+\omega_{2} d-\omega_{3} V O F F-\omega_{4}\left|v_{i}^{0}-v_{i}^{c}\right|-\omega_{5}\left(\tanh \left(\frac{5}{v^{*}}\left(v_{i}^{c}-v^{*}\right) \mathbf{1}_{R^{+}}\left(v_{i}^{c}-v^{*}\right)-3\right)-\tanh (-3)\right)
$$

\section{Results and Discussion}

Various simulation results are presented in Fig. 3, where each plot represents a case with certain initial aircraft states. Black and red circles represent aircraft at time $t=t_{0}$ and $t=t_{0}+\Delta t$, respectively. In these simulations, we used $\Delta t=900 \mathrm{~s}$. The green line represents the freeze horizon. On the bottom left and right of the figures the utilities achieved by the pilots are given together with individual components constituting the utilities. "uETO", "uLOS", "uVOFF", "uEfrt" and "uViolt" represent the utility components corresponding to Estimated Time of Arrival, Loss Of Separation, Vectoring OFF, Effort and Violation of merging point constraint, respectively. Values inside the parenthesis next to each utility component value represent the corresponsing utility weights. Same weights are used in all simulations. The constants in (1) are selected as $t^{*}=2$ (minutes) and $c=2$. Individual utility components are normalized with their maximum possible values during the simulations to obtain order of magnitude comparable utility components. For presentation purposes, the resulting values are multiplied by 100 to give percentage values.

In the figures, $x$ and $v$ next to the aircraft are representing the aircraft distance to the merging point and aircraft velocity, respectively. For example, in Fig. 3-c, the aircraft on the left (first aircraft) starts with a distance of $226 \mathrm{nmi}$ to the merging point with a speed of 350 knots while the other aircraft (second aircraft) has $233 \mathrm{nmi}$ and 394 knots as its initial conditions. When they both observe each other, albeit noisily, the first pilot decides to lower her speed by 3 knots to 347 knots and the second pilot decides to lower her speed by 8 knots to 386 knots. After 15 minutes, these decisions lead the first aircraft to a position where it's $136 \mathrm{nmi}$ away from the merging point while the second aircraft ends up being $133 \mathrm{nmi}$ away. With these final positions and speeds, the first pilot achieves a "-13" utility value, or "happiness index", while the other pilot scores " +6 " points. The individual utility component analysis reveals that although the first pilot achieved higher scores in terms of estimated time of arrival (ETA), effort and constraint violation, her score is less than the other pilot because she is vectored off from landing which decreased her total score by a $100 * 0.4=40$ points. The reason she is vectored off is that both aircraft has approximately equal ETA to the merging point, which creates a potential conflict, but the first aircraft has a little bit higher ETA, which made the air traffic controller decide that it is safer to let the second aircraft continue it's path and vector-off the first aircraft. It is noted that these simulation results are a result of certain assumptions for the initial configurations, vectoring-off criteria, utility function components and merging point constraints. These assumptions can be modified to make the related simulations more realistic. What is emphasized in this work is that once these assumptions are set, based on real data and pilot interviews, the semi net-form game formalism is capable of making predictions on the overall system evolution where human decision plays 
a critical role. The individual parameters in utility functions, such as weights, can also be estimated by using real data via machine learning methods.

The approach presented in this paper can be utilized for flight safety studies such as predicting the percentage of potential collisions, identifying safe separation distance for the aircraft before entering the freeze horizon together with safe speed ranges. In addition, performance predictions such as the percentage of vectored aircraft or average landing times can be obtained. Figure 4 shows the results of an example study where the effect of relaxing the merging point speed constraint on system outcome is investigated. When the merging point speed constraint is relaxed (increased) $20 \%$, the distribution of the commanded speed by the first pilot (v1-c) is more uniform, compared the the default case, where the pilot chooses small speeds more frequently, as expected. The number of vectored aircraft decreases from 211 to 135 , a $36 \%$ drop. This may be due to the fact that since the pilots have more freedom to choose their speeds, they used this extra degree of freedom to prevent vectoring.

\section{Conclusion}

In this paper semi net-form game formalism is implemented to predict a merging and landing scenario, where two aircraft pilots observe each others' states via an ADS-B communication link, while approaching a freeze horizon. Pilots want to arrive the freeze horizon first to gain the first place in landing to save time, but they also consider safety, effort, merging point constraint violation before they make their decision of the new speed command they give to the autopilot. Using certain assumptions about the system configuration and pilot utility functions, a prediction of the system evolution in time is provided. As seen in the simulation results, net-form game formalism provides a powerful analysis tool and can also be used to optimally design the airspace. In addition, net-form game formalism provides a modular, fully probabilistic modeling capacity with reasonable computational cost.

\section{References}

\footnotetext{
${ }^{1}$ Lee, R., Wolpert, D.: Decision Making with Multiple Imperfect Decision Makers, chapter Game Theoretic Modeling of Pilot Behavior during Mid-Air Encounters. Intelligent Systems Reference Library Series. Springer, 2011.

${ }^{2}$ Koller, D., Friedman, N.: Probabilistic graphical models: principles and techniques. MIT Press (2009)

${ }^{3}$ Myerson, R: Game theory. Analysis of conflict, Harvard University Press, Cambridge, MA. (1990).

${ }^{4}$ Camerer, C.F.: Behavioral game theory: experiments in strategic interaction. Princeton University Press (2003)

${ }^{5}$ Crawford, V.P.: Modeling behavior in novel strategic situations via level-k thinking. GAMES 2008. Third World Congress of the Game Theory Society (2008)

${ }^{6}$ McKelvey, R., Palfrey, T.: Quantal Response Equilibria for Normal Form Games, Games and Economic Behavior 10: 638, doi:10.1006/game.1995.1023 (1995)

${ }^{7}$ Neuman, F., Erzberger, H. and Meyn, L. A. : A Fast-Time Simulation Tool for Analysis of Airport Arrival Traffic. NASA Technical Report TP-2004-212283. Ames Research Center, Moffett Field, California, Aug. 2004.
} 

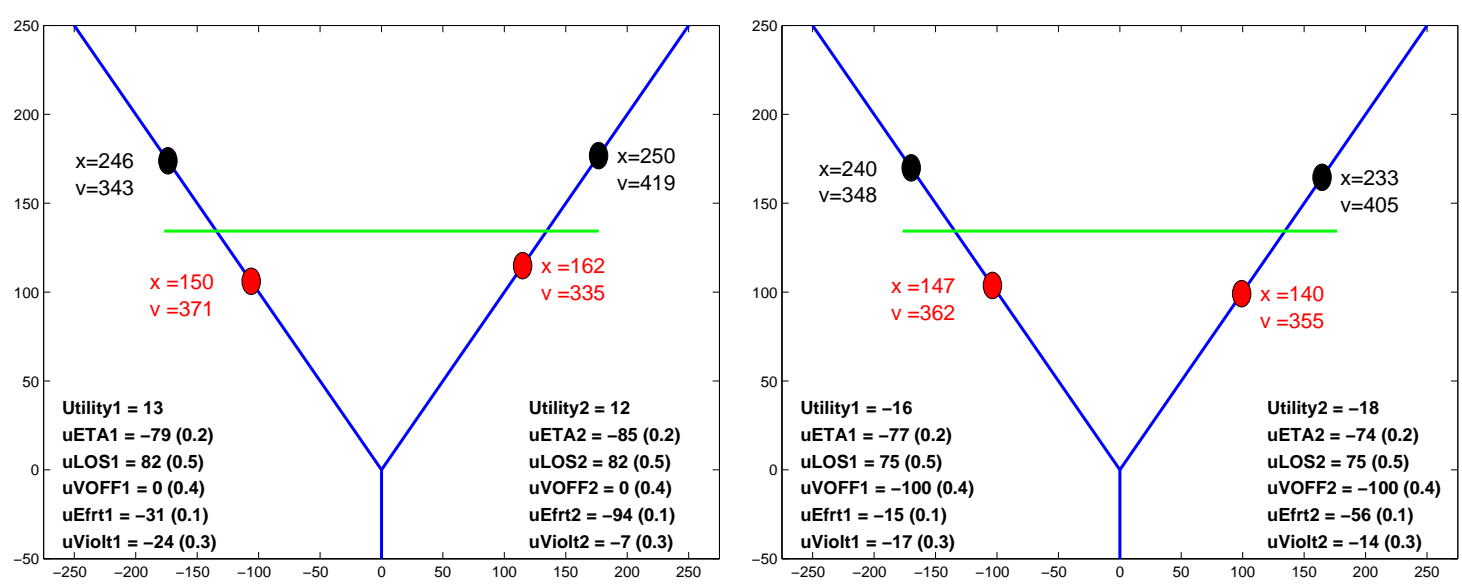

(a) A1: $\{226 \mathrm{nmi}, 343 \mathrm{knots}\}, \mathrm{A} 2:\{250 \mathrm{nmi}, 419 \mathrm{knots}\}$

(b) A1: $\{240 \mathrm{nmi}, 348 \mathrm{knots}\}, \mathrm{A} 2:\{233 \mathrm{nmi}, 405 \mathrm{knots}\}$
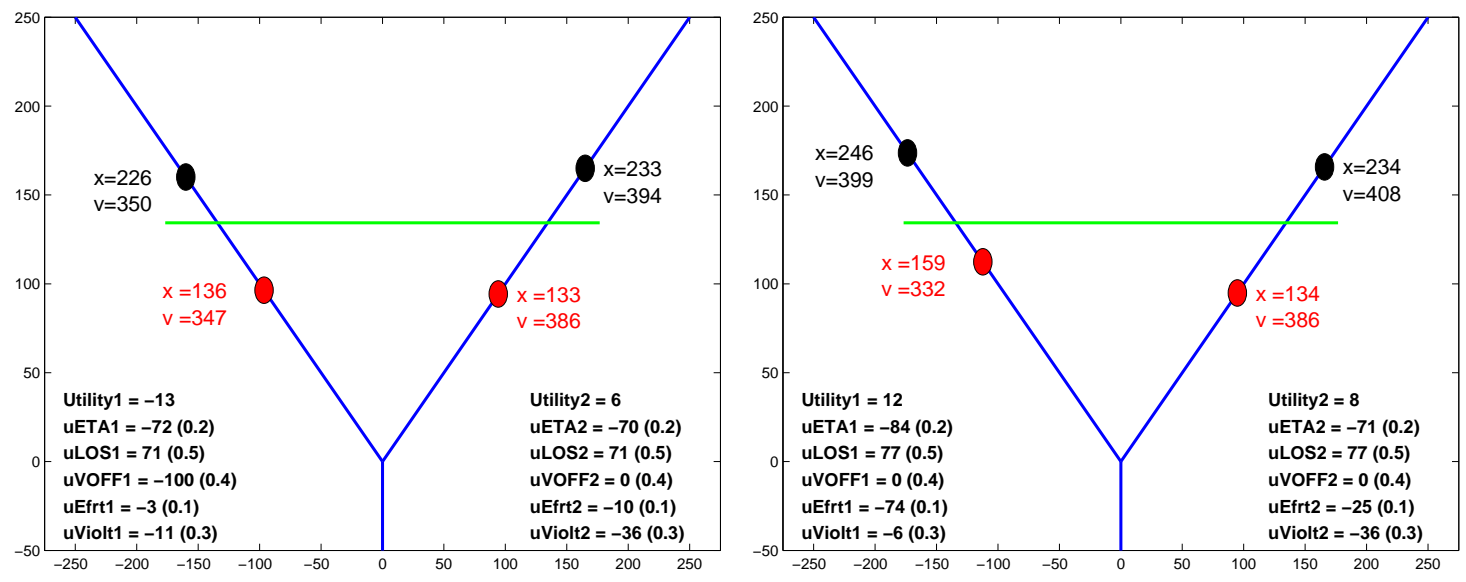

(c) A1: \{226nmi, 350knots $\},$ A2: $\{233 \mathrm{nmi}, 394 \mathrm{knots}\}$

(d) A1: \{246nmi, 399knots $\}, A 2:\{234 \mathrm{nmi}, 408 \mathrm{knots}\}$

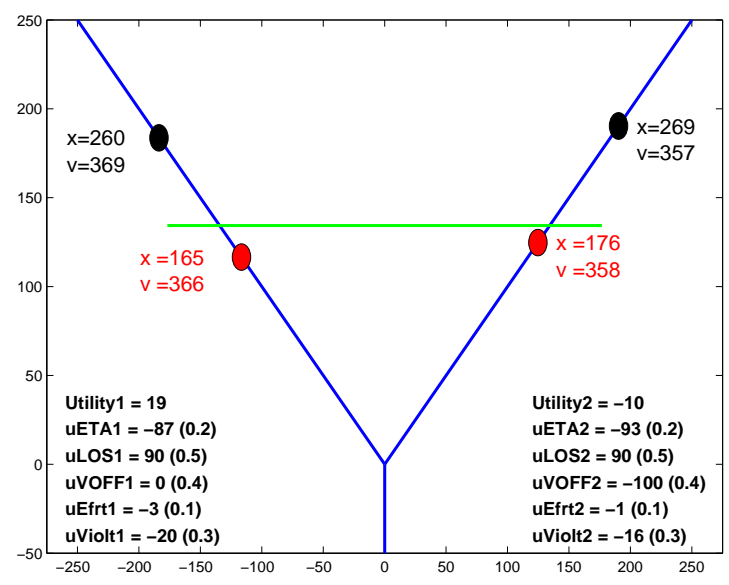

(e) A1: $\{260 \mathrm{nmi}, 369 \mathrm{knots}\}, \mathrm{A} 2:\{269 \mathrm{nmi}, 357 \mathrm{knots}\}$

Figure 3. Various cases with different initial aircraft states

\section{7 of 8}



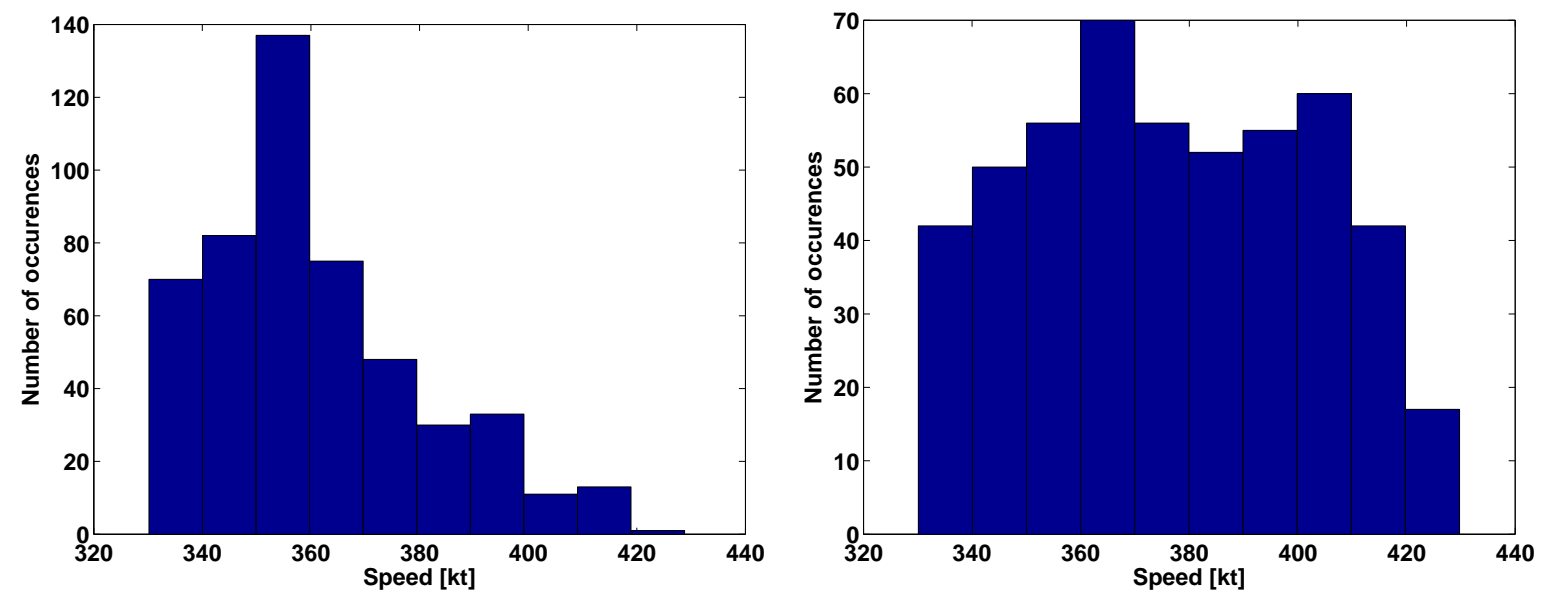

(a) Distribution of the commanded speed for A1, v1-c, (b) Distribution of the commanded speed for A1, v1-c, for default merging point speed constraint

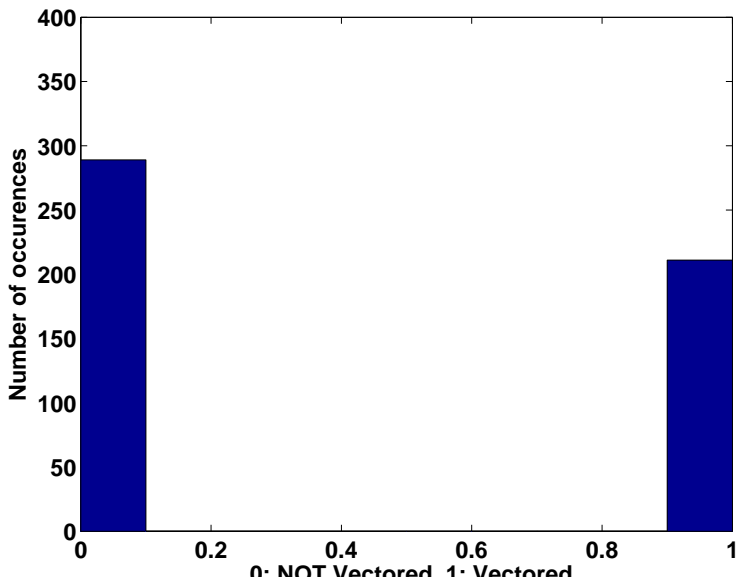

for relaxed merging point speed constraint

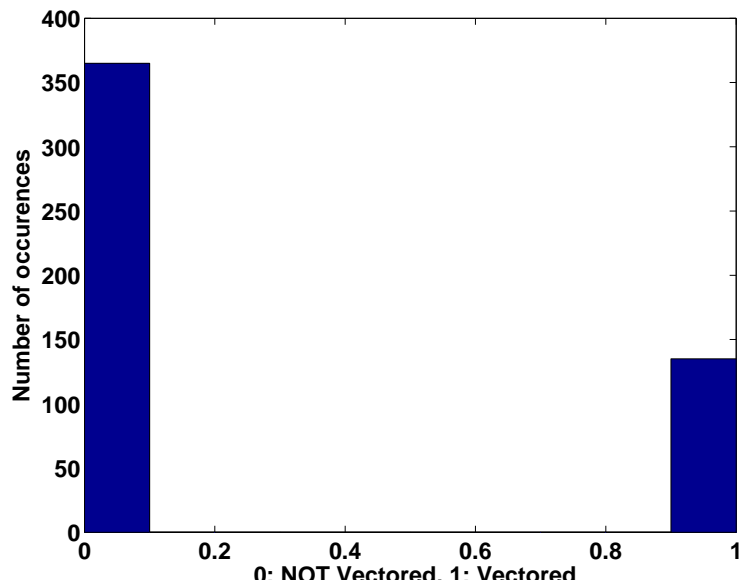

(c) Distribution of the number of vectored aircraft for (d) Distribution of the number of vectored aircraft for default merging point speed constraint

relaxed merging point speed constraint

Figure 4. Effects of relaxing the merging point speed constraint by $20 \%$ 